188 NOMOGRAM OF PEAK EXPIRATORY FLOW RATE VALUES FOR ADOLESCENTS IN A STATE IN SOUTHEAST NIGERIA

Odirichi Andrew, Ifeoma Egbuonu, Ngozi Ojinnaka, Thecla Ezeonu, Obumneme Ezeanosike. Nigeria

\subsection{6/bmjpo-2021-RCPCH.102}

Background Due to the presence of sources of air pollutants (multiple quarry sites and rice mills) in the study area, Peak Expiratory Flow Rate (PEFR) obtained in other parts of the country may not be safely extrapolated to this region. Additionally, the reality of the need to classify asthma exacerbations during emergencies or initial visits where a personal best PEFR is not available has made the development of local PEFR reference values paramount.

Objectives This study was undertaken to develop a nomogram of peak expiratory flow rate values for adolescents in Ebonyi State, Nigeria.

Methods A cross-sectional study was carried out among 970 adolescents from April to June 2018, using a multistage random sampling technique. The PEFR for each subject was determined by the standard miniWright peak flow meter. All data obtained were recorded in a proforma and analysed with Statistical Package for the Social Sciences version 21. The PEFR of the subjects were summarised using mean and standard deviation. Linear regression analysis was used to test the relationship between PEFR and its predictors.

Results The overall mean PEFR value was $332 \pm 83 \mathrm{~L} / \mathrm{min}$. The mean PEFR was $359 \pm 96 \mathrm{~L} / \mathrm{min}$ in males and $312 \pm 64 \mathrm{~L} /$ min in females $(t=9.09, p<0.001)$. The predictive equation was derived as follows:

Males: PEFR $=2 *$ Height $+7.8 *$ Age $+1.7 *$ Weight 152.2 192.9

Females: PEFR $=2 *$ Height $+7.8 *$ Age $+1.7 *$ Weight -

A PEFR nomogram for males and females was created from the equation (a sample for males is represented in table 1).
Conclusions Predicted PEFR values were represented in a nomogram as a reference to the adolescent population in the state and may be used to evaluate PEFR in health facilities in the region.

\section{LEVETIRACETAM AS THE FIRST LINE TREATMENT FOR NEONATAL SEIZURES - A SYSTEMATIC REVIEW AND META-ANALYSIS}

Robert Hooper, Viraraghavan Vadakkencherry Ramaswamy, Rachael Wahid, Prakash Satodia, Adarsh Bhulani. UK

\subsection{6/bmjpo-2021-RCPCH.103}

Background Seizures are the most significant neonatal emergency, with implications on neurodevelopment and mortality. Evidence for the best management of them still remains limited. Phenobarbital is currently the most used drug for neonatal seizure management. The use of Levetiracetam as an alternative is increasing. It is hypothesised to have a better safety profile.

Objectives Assess the effectiveness and safety of levetiracetam when used as the first line treatment of neonatal seizures.

Methods Three electronic databases; MEDLINE, EMBASE, and Web of Science were systematically searched from inception until 20th November 2020. Randomized controlled trials (RCTs) and observational studies that included term and preterm neonates were eligible for inclusion. The primary outcome measure was effectiveness of levetiracetam, defined as seizure cessation within 24 hours of starting treatment. Secondary outcomes included short-term adverse events, mortality before discharge and long-term neurodevelopmental outcomes.

Results 14 studies assessing 1,188 neonates were included. Four were RCTs, three observational trials with phenobarbital as the control arm and seven observational studies of levetiracetam with no control arm. Pooled efficacy of levetiracetam from observational studies was 45\% (95\% CI- 34\%-57\%). Meta-analysis of RCTs evaluating levetiracetam versus phenobarbital showed that both were equally effective [RR (95\%

\begin{tabular}{|c|c|c|c|c|c|c|c|c|c|c|}
\hline \multirow[t]{2}{*}{ Weight (kg) } & \multicolumn{10}{|c|}{ Age in year (Median height in $\mathrm{cm}$ ) } \\
\hline & $10(143)$ & 11 (143) & 12 (148) & 13 (152) & 14 (155) & 15 (159) & $16(163)$ & 17 (165) & $18(165)$ & 19 (165) \\
\hline 20 & 246 & 254 & 271 & 287 & 301 & 317 & 333 & 344 & 352 & 360 \\
\hline 25 & 254 & 262 & 280 & 296 & 310 & 325 & 341 & 353 & 361 & 369 \\
\hline 30 & 263 & 271 & 288 & 304 & 318 & 334 & 350 & 361 & 369 & 377 \\
\hline 35 & 271 & 279 & 297 & 313 & 327 & 342 & 358 & 370 & 378 & 386 \\
\hline 40 & 280 & 288 & 305 & 321 & 335 & 351 & 367 & 378 & 386 & 394 \\
\hline 45 & 288 & 296 & 314 & 330 & 344 & 359 & 375 & 387 & 395 & 403 \\
\hline 50 & 297 & 305 & 322 & 338 & 352 & 368 & 384 & 395 & 403 & 411 \\
\hline 55 & 305 & 313 & 331 & 347 & 361 & 376 & 392 & 404 & 412 & 420 \\
\hline 60 & 314 & 322 & 339 & 355 & 369 & 385 & 401 & 412 & 420 & 428 \\
\hline 65 & 322 & 330 & 348 & 364 & 378 & 393 & 409 & 421 & 429 & 437 \\
\hline 70 & 331 & 339 & 356 & 372 & 386 & 402 & 418 & 429 & 437 & 445 \\
\hline 75 & 339 & 347 & 365 & 381 & 395 & 410 & 426 & 438 & 446 & 454 \\
\hline 80 & 348 & 356 & 373 & 389 & 403 & 419 & 435 & 446 & 454 & 462 \\
\hline 85 & 356 & 364 & 382 & 398 & 412 & 427 & 443 & 455 & 463 & 471 \\
\hline 90 & 365 & 373 & 390 & 406 & 420 & 436 & 452 & 463 & 471 & 479 \\
\hline 95 & 373 & 381 & 399 & 415 & 429 & 444 & 460 & 472 & 480 & 488 \\
\hline
\end{tabular}


CI) - 0.6 (0.3-1.20)] (GRADE - Very low). Levetiracetam resulted in a lower risk of short-term adverse events compared to phenobarbital [RR (95\% CI) - $0.24(0.06-0.92)]$ (GRADE - Moderate).

Conclusions Very low-quality evidence suggests that levetiracetam might not be more effective than phenobarbital. Moderate quality evidence indicates levetiracetam is associated with a lower risk of adverse events.

\section{AN AUDIT OF THE TIME FROM BIRTH TO FIRST MILK FEED IN PRETERM INFANTS LESS THAN 32 WEEKS GESTATION AND OF LOW-BIRTH-WEIGHT INFANTS UNDER $1500 \mathrm{G}$}

Natasha Ramsden. UK

10.1136/bmjpo-2021-RCPCH.104

Background There are many benefits to starting breast milk feeds early for both preterm and low-birth-weight infants including: the immunological benefits of receiving breast milk, potential to establish full enteral feeds earlier and lowering the risk of necrotising enterocolitis.

Objectives Trust guidelines state that all infants should receive colostrum in the first day of life, preferably within the first 6 hours. The objectives of this audit were to evaluate whether this is achieved in infants $<32$ weeks gestation or with birth weights $<1500 \mathrm{~g}$ and to look at the impact of gestation on the time from birth to first milk feed.

Methods Inclusion criteria were infants born $<32$ weeks gestation or with a birthweight of $<1500 \mathrm{~g}$. The length of time from birth to first milk feed in hours (including either colostrum mouth care or first enteral feed) was evaluated on a total of 52 inpatients on the neonatal unit. Data was initially collected in December 2018 on 24 infants. This was followed by interventions including: education of parents and the multidisciplinary team about the importance of the early colostrum administration and collaboration between the neonatal and maternity teams to provide expressing packs to mother's predelivery. Further data was then collected on 28 infants in July 2020, all born during the first wave of the COVID-19 pandemic. The data from 2018 and 2020 were compared and the impact of gestation was analysed by comparing infants born $<27$ weeks and $\geq 27$ weeks.

Results In $100 \%$ of infants in the study the first milk received was human milk (either mother's own milk or donor breast milk) and all infants received their first milk within 96 hours of birth. Overall, 19.2\% of infants received their first milk within 6 hours of birth and $63.5 \%$ within 24 hours. When comparing the 2018 and 2020 data, a similar percentage of infants received their first milk feed in $<24$ hours: $62.5 \%$ in 2018 and $64.3 \%$ in 2020 , and at $<6$ hours: $20.8 \%$ in 2018 and $17.9 \%$ in 2020 . Infants $\geq 27$ weeks received their first milk feed sooner than infants $<27$ weeks. In extreme preterm infants $<27$ weeks, $10 \%$ received their first milk feed in $<6$ hours and $55 \%$ in $<24$ hours. Comparably in preterm infants $\geq 27$ weeks, $25 \%$ received their first milk feed in $<6$ hours and $68.8 \%$ in $<24$ hours. In both groups, $90 \%$ of infants received their first milk feed within 48 hours of birth.

Conclusions The results in 2018 and 2020 were similar, suggesting that the interventions made during this audit cycle had no observed increase in delivery efficiency of the first milk feed. The second cycle of the audit was carried out during the COVID-19 pandemic but even with many restrictions there has been no observed adverse impact on the delivery of the first milk to this group of neonates. Future focus should improve the prompt delivery of the first milk feed for extreme preterm neonates through discussing expressing colostrum with mother's pre-delivery and giving colostrum mouthcare early to these infants.

\section{LUDWIG'S ANGINA-A CASE REPORT}

Anupama Mallappa, Derek Veitch. UK

\subsection{6/bmjpo-2021-RCPCH.105}

Background Ludwig's angina was first reported by Wilhelm Frederick von Ludwig in 1836. It is a severe and quickly spreading cellulitis characterized by significant involvement of the sub-mandibular, sublingual and occasionally sub-mental space. This condition is potentially fatal if not treated aggressively and promptly due to the cellulitis leading to airway compromise. Prior to antibiotics in the middle of the 20th century, mortality rates were more than $50 \%$.

Most cases of Ludwig's angina documented occur more commonly in adult males and secondary to dental infection (70-90\%) with mandibular molars (unilateral or bilateral involvement) as the main cause. When Ludwig's angina occurs in the paediatric population, odontogenic cause is only $50 \%$. Some reported causes include salivary gland infection due to sialadenitis, oral tumour infection and infection following intraoral soft tissues surgery. A precipitating aetiology may be difficult to determine, the most frequently involved microorganism is streptococci and staphylococci species. Diagnosis of the condition is normally made through history and physical examination, other findings include fever and raised white cell count and raised CRP.

Objectives The patient presented here shows a severe case of a Ludwig's angina found in a healthy adolescent male, an age group in which the condition is less common.

Methods electronic case records were used to collect data

Results 13-year-old boy presented with increased facial swelling, drooling, swollen protruding tongue, unable to speak, soft stridor and unable to open mouth.

Had had root canal treatment 7 days prior and had also attended A\&E 2 days previously with fever, pain on swallowing associated with puffiness around cheeks and eyes. He was assessed and sent home on oral antibiotics.

He had no significant past medical history (extraction of 10 deciduous teeth at $7 \mathrm{yrs}$ ).

On presentation to A\&E he had a obstructed airway, maintaining saturations 96\% room air, febrile, he was tachyopneic, tachycardiac(122/min) and hypertensive (113/99 $\mathrm{mmHg}) . \mathrm{He}$ was managed with nebulized adrenaline, dexamethasone and IV antibiotics. Clinical diagnosis was Ludwig's angina

He underwent awake fibro-optic intubation, in theatre he underwent dental extraction LL6 (noted to have copious pus) +UL6, underwent tracheostomy for airway management.

Was transferred to HDU post procedure. CT scan showed fluid collection containing flecks of air within the left parapharyngeal space, extending antero-superiorly to left postnasal space, laterally insinuating between the masseter and left medial pterygoid muscle. Posteriorly, extending to lie just below left bony external acoustic canal. 